\title{
Painlevé solution of an integral equation
}

\author{
Y.Y. Atas, E. Bogomolny \\ Univ. Paris Sud, CNRS, LPTMS, UMR8626, Orsay F-91405, France
}

\begin{abstract}
It is demonstrated that a certain integral equation can be solved using the Painlevé equation of third kind. Inversely, a special solution of this Painlevé equation can be expressed as the ratio of two infinite series of spheroidal functions with known coefficients.
\end{abstract}

\section{Introduction}

We consider an integral equation in a finite interval for unknown function $g(t)$

$$
\int_{-1}^{1} K(|x-t|) g(t) \mathrm{d} t=f(x)
$$

where kernel $K(w)$ has the form

$$
K(w)=w^{\nu} K_{\nu}(\theta w)
$$

Here $K_{\nu}(w)$ is the modified Bessel function of the third kind which obeys the equation

$$
K_{\nu}^{\prime \prime}(w)+\frac{1}{w} K_{\nu}^{\prime}(w)-\left(1+\frac{\nu^{2}}{w^{2}}\right) K_{\nu}(w)=0
$$

and exponentially decreases at large argument

$$
K_{\nu}(w) \underset{w \rightarrow \infty}{\longrightarrow} \sqrt{\frac{\pi}{2 w}} \mathrm{e}^{-w}
$$

At small $w$

$$
K_{\nu}(w) \underset{w \rightarrow 0}{\longrightarrow} 2^{\nu-1} \Gamma(\nu) w^{-\nu}+2^{-\nu-1} \Gamma(-\nu) w^{\nu}+\mathrm{O}\left(w^{2-|\nu|}\right) .
$$

We restrict the discussion to the case $|\nu|<1 / 2$ though certain results could be generalized to all $\nu>-1 / 2$.

Equation (11) with kernel (2) appeared in some physical problems. For example, it describes forced convection heat transfer and contact problems in elasticity (see [1, 2] and references therein). The particular case of (1) with $\nu=0$ and imaginary $\theta$ gives the solution of the wave scattering problem with the Dirichlet boundary conditions at the strip $[-1,1]$ (see e.g. [3]). 
In [1] the solution of this equation has been represented as a series of the spheroidal functions which generalizes the expansion in terms of Mathieu functions for the case $\nu=0$ obtained in [4]. Equation (11) belongs to the class of equations whose particular solutions can be obtained by Latta's method [5] but the resulting equations contain unknown constants. For $\nu=0$ these constants have been explicitly calculated by Myers [6] through a solution of the Painlevé equation of third kind (a concise summary of Myers' result is given in Appendix B of [7]).

The purpose of this note is to show that methods used for $\nu=0$ can be generalized for non-zero $\nu(|\nu|<1 / 2)$ and special solutions of (1) can be obtained from ordinary differential equations (ODE) using the Painlevé III equation more general than the one discussed in [6]. As a by-product we obtain an explicit formula for a solution of the Painlevé III equation as a ratio of two infinite series of spheroidal functions with known coefficients.

The plan of the paper is the following. In Section 2 we use Latta's method to obtain a system of ODE in $t$ variable for two special solutions of (11) corresponding to the right-hand side of (11) equals $\cosh \theta x$ and $\sinh \theta x$. These equations contain certain constants which are determined in Section 3 by deriving the second system of ODE with respect to variable $\theta$. The condition of the compatibility of these two systems of equations leads to the Painlevé III equation for the constants. The limiting behaviour of necessary quantities for small and large $\theta$ are derived in Section 4. Though the discussed methods work only for the above mentioned particular choices of the right-hand side of (1), in Section 5 it is demonstrated that the knowledge of these special solutions permits to investigate more general problems. In Section 6 we show that kernel (2) is a positive-definite function which leads to the boundedness of the discussed Painlevé III solution at positive arguments. For completeness, in Appendix A the series expansion of solutions of integral equation (1) is present and in Appendix B properties of spheroidal functions are briefly described.

\section{First system of equations}

The method of Latta [5] can be applied for integral equations of the form (1) when its kernel, $K(w)$, obeys a differential equation whose coefficients depends linearly on $w$. The kernel $K(w)$ determined in (2) obeys the equation

$$
w K^{\prime \prime}(w)+(1-2 \nu) K^{\prime}(w)-w \theta^{2} K(w)=0
$$

so the application of Latta's method is straightforward.

Let us define the integral operator corresponding to (11)

$$
(\Gamma g)(x) \equiv \int_{-1}^{1} K(|x-t|) g(t) \mathrm{d} t
$$

and its two particular solutions, $g_{c}(t)$ and $g_{s}(t)$, with the right-hand side equals $\cosh (\theta x)$ and $\sinh (\theta x)$

$$
\left(\Gamma g_{c}\right)(x)=\cosh (\theta x), \quad\left(\Gamma g_{s}\right)(x)=\sinh (\theta x)
$$


It is plain that parities of $g_{c, s}(t)$ are fixed: $g_{c}(-t)=g_{c}(t)$ and $g_{s}(-t)=-g_{s}(t)$.

An important ingredient of Latta's method is the uniqueness of the solution: when $f(x)=0$ the only solution of (11) is $g(t)=0$. For kernels like (2) it is known that if $f(x)$ belongs to $L_{2}(-1,1)$ then the solution is unique. A simple way to prove it is to use the expansion into a complete set of functions as in Appendix A.

Changing in (6) variable $w$ to $x-t$, multiplying the resulting expression by $g(t)$, and integrating over $t \in[-1,1]$ gives the following identity (the first Latta equation)

$$
(\Gamma t g)^{\prime \prime}(x)-\theta^{2}(\Gamma t g)(x)=x\left[(\Gamma g)^{\prime \prime}(x)-\theta^{2}(\Gamma g)(x)\right]+(1-2 \nu)(\Gamma g)^{\prime}(x)=0
$$

which permits to calculate $(\Gamma t g)$ when function $f(x) \equiv(\Gamma g)(x)$ is known and obeys the equation $f^{\prime \prime}-\theta^{2} f=0$.

The second Latta equation is obtained by remarking that the kernel in (1) depends only on the difference of arguments. Therefore, it is easy to check that for arbitrary function $y(t)$ such that $y( \pm 1)=0$

$$
(\Gamma y)^{\prime}(x)=\left(\Gamma y^{\prime}\right)(x) .
$$

To apply this equation it is necessary first to determine the behaviour of function $g(t)$ near ends of the strip, $t= \pm 1$. Collecting the most singular terms as it is done in Section 4 or using the representation (A.5) gives

$$
g_{c, s} \underset{t \rightarrow 1}{\longrightarrow} \frac{k_{c, s}}{(1-t)^{\nu+1 / 2}} .
$$

Therefore when $\nu<1 / 2$ functions

$$
y_{c, s}(t)=\left(1-t^{2}\right) g_{c, s}(t)
$$

are zero at the both ends of the strip, $y_{c, s}( \pm 1)=0$ and (10) is valid for them.

Equations (9) and (10) permit to derive ODE for special solutions (8) as follows. By definition

$$
\left(\Gamma g_{c}\right)(x)=\cosh \theta x
$$

From (9) it follows that $y=\left(\Gamma t g_{c}\right)$ obeys the equation

$$
y^{\prime \prime}-\theta^{2} y=(1-2 \nu) \theta \sinh \theta x
$$

whose odd solution is

$$
y \equiv\left(\Gamma t g_{c}\right)(x)=\frac{1-2 \nu}{2} x \cosh \theta x+A \sinh \theta x
$$

where $A$ is a constant independent on $x$.

In exactly the same manner the knowledge of $\Gamma t g_{c}$ permits to calculate $\Gamma t^{2} g_{c}$

$$
\left(\Gamma t^{2} g_{c}\right)(x)=\frac{(1-2 \nu)(3-2 \nu)}{8} x^{2} \cosh \theta x+x \sinh \theta x\left(\frac{1-2 \nu}{2} A-\frac{1-4 \nu^{2}}{8 \theta}\right)+C \cosh \theta x
$$


where $C$ is another constant.

Similarly, with certain constants $B$ and $D$

$$
\left(\Gamma g_{s}\right)(x)=\sinh \theta x, \quad\left(\Gamma t g_{s}\right)(x)=\frac{1-2 \nu}{2} x \sinh \theta x+B \cosh \theta x
$$

and

$$
\left(\Gamma t^{2} g_{s}\right)(x)=\frac{(1-2 \nu)(3-2 \nu)}{8} x^{2} \sinh \theta x+x \cosh \theta x\left(\frac{1-2 \nu}{2} B-\frac{1-4 \nu^{2}}{8 \theta}\right)+D \sinh \theta x
$$

Using (10) one gets

$$
\left(\Gamma\left[\left(1-t^{2}\right) g_{c}\right]^{\prime}\right)(x)=\left(\Gamma\left[\left(1-t^{2}\right) g_{c}\right]\right)^{\prime}(x)
$$

From the above relations it follows that

$$
\begin{gathered}
\left(\Gamma\left[\left(1-t^{2}\right) g_{c}\right]^{\prime}\right)(x)=-\frac{(1-2 \nu)(3-2 \nu)}{8}\left[\theta x^{2} \sinh \theta x+2 x \cosh \theta x\right] \\
-\left(\frac{1-2 \nu}{2} A-\frac{1-4 \nu^{2}}{8 \theta}\right)[\theta x \cosh \theta x+\sinh \theta x]+(1-C) \theta \sinh \theta x .
\end{gathered}
$$

Expressing the right-hand side through $\left(\Gamma t^{2} g_{s}\right),\left(\Gamma t g_{c}\right)$ and $\left(\Gamma g_{s}\right)$ one obtains

$$
\left(\Gamma\left[\left(1-t^{2}\right) g_{c}\right]^{\prime}\right)(x)=-\theta\left(\Gamma t^{2} g_{s}\right)(x)+\left(\theta(B-A)-\frac{3-2 \nu}{2}\right)\left(\Gamma t g_{c}\right)(x)+\rho_{1}\left(\Gamma g_{s}\right)(x)
$$

where $\rho_{1}$ is a certain combination of constants $A, B$, and $C$.

As the solution of (1) is unique, the last equation implies that $g_{c}$ and $g_{s}$ have to obey the following ODE

$$
\left(1-t^{2}\right) g_{c}^{\prime}(t)=\left(\nu+\frac{1}{2}-\rho\right) t g_{c}(t)+\left(\rho_{1}-\theta t^{2}\right) g_{s}(t)
$$

with $\rho=(A-B) \theta+2$.

Repeating these calculations for $g_{s}$ gives another equation

$$
\left(1-t^{2}\right) g_{s}^{\prime}(t)=\left(\nu+\frac{1}{2}+\rho\right) t g_{s}(t)+\left(\rho_{2}-\theta t^{2}\right) g_{c}(t)
$$

Imposing the condition that near the end $t=1$ functions $g_{c}(t)$ and $g_{s}(t)$ have the prescribed singularities (cf. (11))

$$
g_{c}(t) \underset{t \rightarrow 1}{\longrightarrow} \frac{k_{c}}{(1-t)^{\nu+1 / 2}}, \quad g_{s}(t) \underset{t \rightarrow 1}{\longrightarrow} \frac{k_{c} \eta}{(1-t)^{\nu+1 / 2}}
$$

where $\eta$ is determined from the limit

$$
\eta \equiv \frac{k_{s}}{k_{c}}=\lim _{t \rightarrow 1} \frac{g_{s}(t)}{g_{c}(t)}
$$

one fixes constants $\rho_{1}$ and $\rho_{2}$. 
Finally, we conclude that for $|\nu|<1 / 2$ functions $g_{c}(t)$ and $g_{s}(t)$ obey the system of ODE

$$
\frac{\partial}{\partial t}\left(\begin{array}{l}
g_{c} \\
g_{s}
\end{array}\right)=M\left(\begin{array}{l}
g_{c} \\
g_{s}
\end{array}\right)
$$

with the following $2 \times 2$ matrix $M$

$$
M=\left(\begin{array}{cc}
0 & \theta \\
\theta & 0
\end{array}\right)+\frac{1}{1-t^{2}}\left(\begin{array}{cc}
t(\nu+1 / 2-\rho) & \frac{1}{\eta}(\nu+1 / 2+\rho) \\
\eta(\nu+1 / 2-\rho) & t(\nu+1 / 2+\rho)
\end{array}\right) .
$$

When $\nu=0$ these equations coincide with the ones in [5].

For the further use we rewrite the system (26) in the form

$$
\begin{aligned}
\frac{\partial}{\partial t}\left[g_{c}-\frac{t}{\eta} g_{s}\right] & =-\frac{\theta t}{\eta} g_{c}+\left(\frac{\nu-\frac{1}{2}+\rho}{\eta}+\theta\right) g_{s}, \\
\frac{\partial}{\partial t}\left[g_{s}-t \eta g_{c}\right] & =-\theta t \eta g_{s}+\left(\eta\left(\nu-\frac{1}{2}-\rho\right)+\theta\right) g_{c} .
\end{aligned}
$$

The terms in the square brackets are zero when $t= \pm 1$ and this system can be obtained directly by using (10).

\section{Second system of equations}

To find constants $\rho$ and $\eta$ we use scaling arguments similar but different to the ones discussed in [6].

Functions $g_{c}$ and $g_{s}$ in (8) depend on $t$ and $\theta, g_{c, s}=g_{c, s}(t, \theta)$. We are interesting in finding equations governing the evolution of these function with changing $\theta$.

Consider instead of system (8) a more general system of equations

$$
\begin{aligned}
\Gamma G_{c} & \equiv \int_{L_{1}}^{L_{2}}|x-t|^{\nu} K_{\nu}(k|x-t|) G_{c}(t) \mathrm{d} t=\cosh \left[k\left(x-\frac{1}{2}\left(L_{1}+L_{2}\right)\right)\right], \\
\Gamma G_{s} & \equiv \int_{L_{1}}^{L_{2}}|x-t|^{\nu} K_{\nu}(k|x-t|) G_{s}(t) \mathrm{d} t=\sinh \left[k\left(x-\frac{1}{2}\left(L_{1}+L_{2}\right)\right)\right]
\end{aligned}
$$

Changing the variable

$$
t=\frac{1}{2}\left(L_{1}+L_{2}\right)+\frac{1}{2}\left(L_{2}-L_{1}\right) y
$$

together with the similar change of $x$ it is straighforward to check that functions $G_{c}(y)$ and $G_{s}(y)$ are expressed through $g_{c}(t)$ and $g_{s}(t)$ defined in (8) as follows

$$
G_{c, s}(y)=\left(\frac{2}{L_{2}-L_{1}}\right)^{\nu+1} g_{c, s}(z, \theta)
$$

where

$$
z=\frac{2}{L_{2}-L_{1}} y-\frac{L_{2}+L_{1}}{L_{2}-L_{1}}, \quad \theta=\frac{1}{2} k\left(L_{2}-L_{1}\right) .
$$

Consider two functions

$$
\Psi_{1}(y)=G_{s}(y)+\eta G_{c}(y), \quad \Psi_{2}(y)=G_{s}(y)-\eta G_{c}(y)
$$


where $\eta$ is the same as in (25). By construction $\Psi_{1}\left(L_{1}\right)=0$ and $\Psi_{2}\left(L_{2}\right)=0$.

From (29) it follows that

$$
\Gamma \Psi_{1,2}=\Gamma G_{s} \pm \eta \Gamma G_{c}=\sinh \left(k\left(x-\frac{1}{2}\left(L_{1}+L_{2}\right)\right) \pm \eta \cosh \left(k\left(x-\frac{1}{2}\left(L_{1}+L_{2}\right)\right)\right.\right.
$$

As $\Psi_{i}\left(L_{i}\right)=0$, the differentiation over $L_{i}$ of these equations gives

$$
\frac{\partial}{\partial L_{i}}\left(\Gamma \Psi_{i}\right)=\Gamma\left(\frac{\partial}{\partial L_{i}} \Psi_{i}\right), \quad i=1,2
$$

Using the uniqueness of the solutions, after simple algebra we obtain

$$
\begin{aligned}
& \frac{\partial G_{s}}{\partial L_{1}}+\eta \frac{\partial G_{c}}{\partial L_{1}}=-\frac{\theta}{L_{2}-L_{1}}\left(G_{c}+\eta G_{s}\right), \\
& \frac{\partial G_{s}}{\partial L_{2}}-\eta \frac{\partial G_{c}}{\partial L_{2}}=-\frac{\theta}{L_{2}-L_{1}}\left(G_{c}-\eta G_{s}\right) .
\end{aligned}
$$

Performing the calculations one finds

$$
\frac{\partial G_{c, s}}{\partial L_{i}}=\frac{(-1)^{i+1}}{L_{2}-L_{1}}\left(\frac{2}{L_{2}-L_{1}}\right)^{\nu+1}\left((1+\nu) g_{c, s}+\left(z+(-1)^{i}\right) \frac{\partial g_{c, s}}{\partial z}-\theta \frac{\partial g_{c, s}}{\partial \theta}\right)
$$

where $i=1$ for $G_{c}$ and $i=2$ for $G_{s}$.

Combining all terms together one obtains that (36) take the the form

$$
\begin{aligned}
& \theta \eta \frac{\partial g_{c}}{\partial \theta}=-\left(\frac{\partial g_{s}}{\partial z}-\eta z \frac{\partial g_{c}}{\partial z}\right)+(\theta+\eta(1+\nu)) g_{c} \\
& \theta \frac{\partial g_{s}}{\partial \theta}=-\left(z \frac{\partial g_{s}}{\partial z}-\eta \frac{\partial g_{c}}{\partial z}\right)+(\theta \eta+(1+\nu)) g_{s}
\end{aligned}
$$

Using (28) we conclude that functions $g_{c, s}(t, \theta)$ obey the following system of equations

$$
\frac{\partial}{\partial \theta}\left(\begin{array}{l}
g_{c} \\
g_{s}
\end{array}\right)=N\left(\begin{array}{l}
g_{c} \\
g_{s}
\end{array}\right)
$$

where matrix $N$ has the form

$$
N=\left(\begin{array}{cc}
\frac{1 / 2+\rho}{\theta} & t \\
t & \frac{1 / 2-\rho}{\theta}
\end{array}\right) .
$$

The condition of compatibility of systems of equations (27) and (39) gives the equations of zero curvature for matrices $N$ and $M$

$$
\frac{\partial}{\partial t} N-\frac{\partial}{\partial \theta} M=M N-N M
$$

Direct calculations prove that this equation will be valid provided $\eta=\eta(\theta)$ and $\rho=\rho(\theta)$ fulfil the equations

$$
\rho=\frac{\theta}{2 \eta}\left(1-\eta^{\prime}-\eta^{2}\right)
$$


and

$$
\rho^{\prime}=\frac{\nu+1 / 2+\rho}{\eta}-(\nu+1 / 2-\rho) \eta
$$

Substituting here the previous equation one finds that $\eta$ has to obey equation

$$
\frac{\mathrm{d}^{2} \eta}{\mathrm{d} \theta^{2}}=\eta^{-1}\left(\frac{\mathrm{d} \eta}{\mathrm{d} \theta}\right)^{2}-\theta^{-1} \frac{\mathrm{d} \eta}{\mathrm{d} \theta}-\frac{2 \nu\left(1-\eta^{2}\right)}{\theta}+\eta^{3}-\frac{1}{\eta} .
$$

This equation is a particular form of Painlevé III equation which has been discussed in [10].

From the results of Appendix A and Appendix B it follows that functions $g_{c}(\cos \gamma)$ and $g_{s}(\cos \gamma)$ can be written as the following series of the spheroidal functions

$$
\begin{aligned}
& g_{c}(\cos \gamma)=\frac{1}{(\sin \gamma)^{2 \nu+1}} \sum_{m=0}^{\infty} \mu_{2 m} \tilde{X}_{2 m}(0) Y_{2 m}(\gamma), \\
& g_{s}(\cos \gamma)=\frac{1}{(\sin \gamma)^{2 \nu+1}} \sum_{m=0}^{\infty} \mu_{2 m+1} \tilde{X}_{2 m+1}(0) Y_{2 m+1}(\gamma)
\end{aligned}
$$

where $Y_{m}(\cos \gamma)$ are defined in (B.1), $X_{m}(\xi)$ in (B.18), and $\mu_{n}$ in (A.24).

Function $\eta$ in (25) which is a solution of the Painlevé III equation is given as the ratio of the above series

$$
\eta=\frac{\sum_{m=0}^{\infty} \mu_{2 m+1} \tilde{X}_{2 m+1}(0) Y_{2 m+1}(0)}{\sum_{m=0}^{\infty} \mu_{2 m} \tilde{X}_{2 m}(0) Y_{2 m}(0)} .
$$

\section{Limiting behaviour}

In order to use the equations derived in the previous Sections it is necessary to know the behaviour of $g_{c}(t)$ and $g_{s}(t)$ defined in (8) in the limit of small $\theta$ and (or) large $\theta$.

From (5) it follows that at small $\theta$

$$
K(w) \underset{\theta \rightarrow 0}{\longrightarrow} \theta^{-\nu} \frac{\Gamma(\nu)}{2^{1-\nu}}+w^{2 \nu} \theta^{\nu} \frac{\Gamma(-\nu)}{2^{1+\nu}} .
$$

Therefore, to find the necessary solutions of (8) when $\theta \rightarrow 0$ it is necessary first to solve the equation

$$
\int_{-1}^{1}|x-t|^{2 \nu} g(t) \mathrm{d} t=f(x)
$$

when function $f(x)$ equals 1 or $x$.

Though the general solution of such equation is known (see e.g. [12]), solutions with $f(x)=1$ and $f(x)=x$ can easily be obtained by the direct application of Latta's method [5]. After a simple algebra one finds that the solutions of equations

$$
\int_{-1}^{1}|x-t|^{2 \nu} g_{0}(t) \mathrm{d} t=1, \quad \int_{-1}^{1}|x-t|^{2 \nu} g_{1}(t) \mathrm{d} t=x
$$

are

$$
g_{0}(t)=\frac{\cos (\pi \nu)}{\pi\left(1-t^{2}\right)^{\nu+1 / 2}}, \quad g_{1}(t)=-\frac{\cos (\pi \nu) t}{2 \pi \nu\left(1-t^{2}\right)^{\nu+1 / 2}} .
$$


Painlevé solution of an integral equation

From (48) we obtain that

$$
g_{c}(t) \underset{\theta \rightarrow 0}{\longrightarrow} \mu_{c} g_{0}(t), \quad g_{s}(t) \underset{\theta \rightarrow 0}{\longrightarrow} \mu_{s} g_{1}(t)
$$

where constants $\mu_{c}$ and $\mu_{s}$ are determined from the conditions

$$
\mu_{c}\left(\theta^{\nu} \frac{\Gamma(-\nu)}{2^{1+\nu}}+\theta^{-\nu} \frac{\cos (\pi \nu) \Gamma(1 / 2-\nu) \Gamma(\nu)}{\sqrt{\pi} 2^{1-\nu} \Gamma(1-\nu)}\right)=1, \quad \mu_{s} \theta^{\nu} \frac{\Gamma(-\nu)}{2^{1+\nu}}=\theta .
$$

From these relations it follows that

$$
\eta(\theta) \underset{\theta \rightarrow 0}{\longrightarrow}-\frac{\mu_{s}}{2 \nu \mu_{c}}=-\theta^{1-2 \nu} \frac{\cos (\pi \nu) \Gamma(1 / 2-\nu) \Gamma(\nu)}{\sqrt{\pi} 2^{1-2 \nu} \nu \Gamma(1-\nu) \Gamma(-\nu)}-\frac{\theta}{2 \nu} .
$$

Using standard formulae for the $\Gamma$-function

$$
\Gamma(2 \nu)=\frac{2^{2 \nu-1}}{\sqrt{\pi}} \Gamma(\nu) \Gamma(\nu+1 / 2), \quad \Gamma(1 / 2+\nu) \Gamma(1 / 2-\nu)=\frac{\pi}{\cos (\pi \nu)},
$$

one gets that the limiting behaviour of $\eta(\theta)$ at small $\theta$ is

$$
\eta(\theta) \underset{\theta \rightarrow 0}{\longrightarrow} B(2 \theta)^{1-2 \nu}-\frac{\theta}{2 \nu}=\left\{\begin{array}{cc}
B(2 \theta)^{1-2 \nu}, & 0<\nu<1 / 2 \\
-\theta /(2 \nu), & -1 / 2<\nu<0
\end{array}\right.
$$

where

$$
B=2^{-3(1-2 \nu)} \frac{\Gamma^{2}(\nu)}{\Gamma^{2}(1-\nu) \Gamma(2 \nu)} .
$$

To find the behaviour of solutions when $\theta \rightarrow \infty$ it is convenient to consider instead of functions $g_{c, s}$ (8) a solution $g_{-}(v)$ corresponding to the integral equation

$$
\int_{-1}^{1}|u-v|^{\nu} K_{\nu}(\theta|u-v|) g_{-}(v) \mathrm{d} v=\mathrm{e}^{-\theta u}
$$

To find asymptotic behaviour of $g_{-}$for large $\theta$ we first consider the equation

$$
\int_{0}^{\infty}|u-v|^{\nu} K_{\nu}(|u-v|) g_{0}(v) \mathrm{d} v=\mathrm{e}^{-u}
$$

Its solution can be calculated either by the Wiener-Hopf method [13] using known Fourier transform of the kernel

$$
\int_{-\infty}^{\infty} \mathrm{e}^{\mathrm{i} p x} K_{\nu}(\theta x) x^{\nu} \mathrm{d} x=\frac{(2 \theta)^{\nu} \sqrt{\pi} \Gamma(\nu+1 / 2)}{\left(p^{2}+\theta^{2}\right)^{\nu+1 / 2}}
$$

or by expansion formulae analogous to the ones described in Appendix A but for half-line integration 15.

$$
\begin{aligned}
& n ! \int_{0}^{\infty}|x-t|^{\nu} K_{\nu}\left(\frac{1}{2}|x-t|\right) t^{-\nu-1 / 2} \mathrm{e}^{-t / 2} L_{n}^{-\nu-1 / 2}(t) \mathrm{d} t \\
& =\sqrt{\pi} \Gamma(\nu+1 / 2) \Gamma(n+1 / 2-\nu) \mathrm{e}^{-x / 2} L_{n}^{-\nu-1 / 2}(x)
\end{aligned}
$$


where $L_{n}^{\lambda}(x)$ with $n=0,1, \ldots$ are the Laguerre polynomials.

One gets

$$
g_{0}(v)=C_{\nu} v^{-\nu-1 / 2} \mathrm{e}^{-v}, \quad C_{\nu}=\frac{\sqrt{2}}{\pi^{3 / 2}} \cos \pi \nu .
$$

Using this solution it is easy to show that the dominant approximation for the solution of (58) when $\theta \rightarrow \infty$ has the form

$$
g_{-}^{(0)}(t)=C_{\nu} \sqrt{\theta}(1+t)^{-\nu-1 / 2} \mathrm{e}^{-t \theta} .
$$

Straightforward transformations give

$$
\int_{-1}^{1}|x-t|^{\nu} K_{\nu}(\theta|x-t|) g_{-}^{(0)}(t) \mathrm{d} t=F_{1}(x)-F_{2}(x)
$$

where

$$
F_{1}(x)=\int_{-1}^{\infty}|x-t|^{\nu} K_{\nu}(\theta|x-t|) g_{-}^{(0)}(t) \mathrm{d} t, F_{2}(x)=\int_{1}^{\infty}|x-t|^{\nu} K_{\nu}(\theta|x-t|) g_{-}^{(0)}(t) \mathrm{d} t .
$$

Changing the variable in the first integral as $t=-1+v / \theta$ and using (59) leads to

$$
F_{1}(x)=\mathrm{e}^{-\theta x} \text {. }
$$

In the integral for $F_{2}(x)$ it is convenient to put $t=1+s / \theta$. It this way one obtains

$$
F_{2}(x)=\theta^{-\nu-1 / 2} C_{\nu} \int_{0}^{\infty}\left(2+\frac{s}{\theta}\right)^{-\nu-1 / 2}(s+\theta(1-x))^{\nu} K_{\nu}(s+\theta(1-x)) \mathrm{e}^{-\theta-s} \mathrm{~d} s .
$$

When $\theta$ is large one can drop the term $s / \theta$ in the first bracket and use the asymptotic form of the $K_{\nu}$ function (4)

$$
F_{2}(x) \underset{\theta \rightarrow \infty}{\longrightarrow} \theta^{-\nu-1 / 2} 2^{-\nu-1} \sqrt{\pi} \mathrm{e}^{-2 \theta+\theta x} C_{\nu} \int_{0}^{\infty}(s+\theta(1-x))^{\nu-1 / 2} \mathrm{e}^{-2 s} \mathrm{~d} s .
$$

This formula means that close to right-hand side, $x=1$, the additional contribution related with the integration in finite limits is

$$
F_{2}(x) \approx \theta^{-\nu-1 / 2} 2^{-\nu-1} \sqrt{\pi} \mathrm{e}^{-2 \theta+\theta x} C_{\nu} \int_{0}^{\infty} s^{\nu-1 / 2} \mathrm{e}^{-2 s} \mathrm{~d} s=\mathrm{e}^{\theta x} \delta
$$

where

$$
\delta=\frac{1}{2 \pi} \theta^{-\nu-1 / 2} 2^{-2 \nu} \cos (\pi \nu) \mathrm{e}^{-2 \theta} \Gamma(\nu+1 / 2) .
$$

Using (58) it is obvious that to cancel contribution $F_{2}(x)$ it is necessary to modify the approximation (63) as follows

$$
g_{-}(t) \underset{\theta \rightarrow \infty}{\longrightarrow} C_{\nu} \sqrt{\theta}\left[(1+t)^{-\nu-1 / 2} \mathrm{e}^{-t \theta}+\delta(1-t)^{-\nu-1 / 2} \mathrm{e}^{t \theta}\right] .
$$

This function consists of two terms. The first dominates close to $t=-1$ and the second near $t=1$. According to (25) function $\eta(\theta)$ is determined from the limit

$$
\eta(\theta)=\lim _{t \rightarrow 1} \frac{g_{-}(-t)-g_{-}(t)}{g_{-}(-t)+g_{-}(t)}
$$

Using (71) we find that

$$
\eta(\theta) \underset{\theta \rightarrow \infty}{\longrightarrow} 1-\frac{\cos (\pi \nu)}{\pi} \Gamma(\nu+1 / 2) 2^{-2 \nu} \theta^{-\nu-1 / 2} \mathrm{e}^{-2 \theta} .
$$




\section{Embedding formulae}

In the previous Sections the integral equation (1) has been transformed into two systems of ODE (26), (27) and (39), (40) but only for very special right-hand sides of this equation, $f(x)=\cosh (\theta x)$ and $f(x)=\sinh (\theta x)$ (cf. (8) ). Nevertheless, the knowledge of these two special solutions permits to find solutions of (11) for more general cases [7], [8].

Let us consider the equation of the form

$$
(\Gamma g)(x)=\mathrm{e}^{-\theta z x}
$$

with the same kernel as in (2). To find its solution, $g(t)$, it is convenient to define symmetric and antisymmetric combinations of $g_{c, s}$

$$
g_{ \pm}(t)=g_{c}(t) \pm g_{s}(t)
$$

We look for solution of (74) in the form

$$
\Psi(t)=g(t)+a_{+} g_{+}(t)+a_{-} g_{-}(t)
$$

where $a_{+}$and $a_{-}$are two constants independent on $t$ which have to be determined from two conditions

$$
\lim _{t \rightarrow \pm 1} \Psi(t)=0
$$

The explicit form of these constants will be presented later (see (89)) .

Assuming that (177) is fulfilled, one concludes as in Section 2 that

$$
(\Gamma \Psi(x))^{\prime}=\left(\Gamma \Psi^{\prime}\right)(x)
$$

The right-hand side of this equation is calculated from the definition of $\Psi$ and

$$
(\Gamma \Psi(x))^{\prime}=-\theta z \mathrm{e}^{-\theta z x}+\theta a_{+} \mathrm{e}^{\theta x}-\theta a_{-} \mathrm{e}^{-\theta x}=\left(\Gamma\left[-z \theta g+a_{+} \theta g_{+}-a_{-} \theta g_{-}\right]\right)(x) \text {. }
$$

Due to the uniqueness of the solution, $\Psi(t)$ obeys the equation

$$
\Psi^{\prime}(t)=-z \theta\left(\Psi(t)-a_{+} g_{+}(t)-a_{-} g_{-}(t)\right)+a_{+} \theta g_{+}(t)+a_{-} \theta g_{-}(t)
$$

which is equivalent to

$$
\theta^{-1} \Psi^{\prime}(t)+z \Psi(t)=(z+1) a_{+} g_{+}(t)+(z-1) a_{-} g_{-}(t)
$$

whose solution is straightforward if constants $a_{ \pm}$are known.

To find them one can proceed as follows [8]. Let us calculate the following quantities

$$
\left(g_{ \pm} \Gamma \Psi\right) \equiv \int_{-1}^{1} \int_{-1}^{1} g_{ \pm}(x) K(x-t) \Psi(t) \mathrm{d} t \mathrm{~d} x
$$


by two different methods, the first by applying the operator $\Gamma$ on $g_{ \pm}$and the second by applying it on $\Psi$. In such a manner one gets

$$
\int_{-1}^{1} \Psi(t) \mathrm{e}^{ \pm \theta t} \mathrm{~d} t=\int_{-1}^{1} g_{ \pm}(t)\left(\mathrm{e}^{-z \theta t}+a_{+} \mathrm{e}^{\theta t}+a_{-} \mathrm{e}^{-\theta t}\right) \mathrm{d} t .
$$

Introduce the Laplace transform of functions $g_{c, s}$

$$
\hat{G}_{c}(p)=\int_{-1}^{1} g_{c}(t) \mathrm{e}^{p \theta t} \mathrm{~d} t, \quad \hat{G}_{s}(p)=\int_{-1}^{1} g_{s}(t) \mathrm{e}^{p \theta t} \mathrm{~d} t .
$$

Due to the symmetry properties of $g_{c, s}, \hat{G}_{c}(-p)=\hat{G}_{c}(p)$ and $\hat{G}_{s}(-p)=-\hat{G}_{s}(p)$.

Using (84) we obtain

$$
\begin{aligned}
& \int_{-1}^{1} \Psi(t) \mathrm{e}^{\theta t} \mathrm{~d} t=G(-z)+a_{+} G(1)+a_{-} G(-1), \\
& \int_{-1}^{1} \Psi(t) \mathrm{e}^{-\theta t} \mathrm{~d} t=G(z)+a_{+} G(-1)+a_{-} G(1)
\end{aligned}
$$

where $G(p)=\hat{G}_{c}(p)+\hat{G}_{s}(p)$.

On the other hand, multiplying (81) by $\mathrm{e}^{ \pm \theta t}$, integrating the results from -1 to 1 , and taking into account that $\Psi( \pm 1)=0$ one finds

$$
\begin{aligned}
& (z-1) \int_{-1}^{1} \mathrm{e}^{\theta t} \Psi(t) \mathrm{d} t=(z+1) a_{+} G(1)+(z-1) a_{-} G(-1), \\
& (z+1) \int_{-1}^{1} \mathrm{e}^{-\theta t} \Psi(t) \mathrm{d} t=(z+1) a_{+} G(-1)+(z-1) a_{-} G(1) .
\end{aligned}
$$

These and previous equations permits to calculate constants $a_{ \pm}$

$$
a_{ \pm}=-\frac{(1 \mp z) G(\mp z)}{2 G(1)}
$$

Combining the above formulae one finds that (81) takes the form

$$
\theta^{-1} \Psi^{\prime}(t)+z \Psi(t)=\frac{z^{2}-1}{G(1)}\left[\hat{G}_{c}(z) g_{s}(t)-\hat{G}_{s}(z) g_{c}(t)\right]
$$

whose solution obeying $\Psi( \pm 1)=0$ is

$$
\Psi(t)=\frac{\left(z^{2}-1\right) \theta}{G(1)} \mathrm{e}^{-z \theta t}\left[\hat{G}_{c}(z) \int_{-1}^{t} g_{s}(y) \mathrm{e}^{z \theta y} \mathrm{~d} y-\hat{G}_{s}(z) \int_{-1}^{t} g_{s}(y) \mathrm{e}^{z \theta y} \mathrm{~d} y\right] .
$$

The above formulae relate the general plane-wave solution (74) to two special cases corresponding to $z= \pm 1$. Such formulae are called embedding formulae in the theory of diffraction and can be derived in more general settings (see e.g. 14] and references therein). 


\section{Positivity relations}

Equation (91) represents the solution of (74). As for usual diffraction problems it is physically clear (and can be proved e.g. by using the series expansions as in Appendix A) that such solution exist for all $\theta>0$ and any $z$ but (91) contains $G(1)$ in the numerator and in order to the solution remains finite it is necessary that this quantity is always non-zero.

By the construction $G(1)$ is the Laplace transform of the sum $g_{c}+g_{s}$

$$
G(1)=\int_{-1}^{1}\left(g_{c}(t)+g_{s}(t)\right) \mathrm{e}^{\theta t} \mathrm{~d} t=\int_{-1}^{1} g_{c}(t) \cosh (\theta t) \mathrm{d} t+\int_{-1}^{1} g_{s}(t) \sinh (\theta t) \mathrm{d} t .
$$

By definition, $g_{c}(t)$ and $g_{s}(t)$ are solutions of (요 thus

$$
\cosh (\theta x)=\left(\Gamma g_{c}\right)(x), \quad \sinh (\theta x)=\left(\Gamma g_{s}\right)(x)
$$

and

$$
G(1)=\int_{-1}^{1} \mathrm{~d} t \int_{-1}^{1} g_{c}(t) K(|x-t|) g_{c}(x) \mathrm{d} x+\int_{-1}^{1} \mathrm{~d} t \int_{-1}^{1} g_{s}(t) K(|x-t|) g_{s}(x) \mathrm{d} x
$$

with the kernel $K(w)$ defined in (2). An important property of such kernel is that its Fourier transform, $\hat{K}(p)$, presented (60) is strictly positive for all $p$ and $\nu>-1 / 2$. It means that function $K(w)$ is a positive definite function and the double integral

$$
\int_{-1}^{1} \mathrm{~d} t \int_{-1}^{1} f(t) K(|x-t|) f(x) \mathrm{d} x \equiv \frac{1}{2 \pi} \int_{-\infty}^{\infty}\left|\int_{-1}^{1} f(t) \mathrm{e}^{\mathrm{i} p t} \mathrm{~d} t\right|^{2} \hat{K}(p) \mathrm{d} p
$$

is positive for any function $f(x)$. Therefore for all $\theta>0$

$$
G(1)>0
$$

and the solution (91) is finite for all $z$.

The same positivity condition permits also to establish the positivity and finiteness

of $\eta(\theta)$. Formally, this function is defined as the limit when $t \rightarrow 1$ of the ratio of two solutions (25) and it is not evident that it remains finite for all $\theta>0$.

Let us define two functions

$$
\hat{F}_{c}(p)=\int_{-1}^{1} t g_{c}(t) \mathrm{e}^{p \theta t} \mathrm{~d} t, \quad \hat{F}_{s}(p)=\int_{-1}^{1} t g_{s}(t) \mathrm{e}^{p \theta t} \mathrm{~d} t
$$

which differ from (84) by factor $t$ in the integrand.

Multiplying (28) by $\mathrm{e}^{z \theta t}$ and integrating the both parts over the interval $[-1,1]$ one finds

$$
\begin{aligned}
& {\left[\frac{\nu-1 / 2-\rho}{\theta}+\frac{1}{\eta}\right] \hat{G}_{c}(z)+\frac{z}{\eta} \hat{G}_{s}(z)=z \hat{F}_{c}(z)+\hat{F}_{s}(z),} \\
& {\left[\frac{\nu-1 / 2+\rho}{\theta}+\eta\right] \hat{G}_{s}(z)+z \eta \hat{G}_{c}(z)=\hat{F}_{c}(z)+z \hat{F}_{s}(z) .}
\end{aligned}
$$


From (39) and (40) it follows that

$$
\begin{aligned}
& \frac{\partial}{\partial \theta} \hat{G}_{c}(z)-\frac{1 / 2+\rho}{\theta} \hat{G}_{c}(z)=\hat{F}_{s}(z)+z \hat{F}_{c}(z), \\
& \frac{\partial}{\partial \theta} \hat{G}_{s}(z)-\frac{1 / 2-\rho}{\theta} \hat{G}_{s}(z)=\hat{F}_{c}(z)+z \hat{F}_{s}(z) .
\end{aligned}
$$

Combining these relations one concludes that

$$
\frac{\partial}{\partial \theta} \hat{G}_{c}(z)-\left[\frac{\nu}{\theta}+\frac{1}{\eta}\right] \hat{G}_{c}(z)=\frac{z}{\eta} \hat{G}_{s}(z), \quad \frac{\partial}{\partial \theta} \hat{G}_{s}(z)-\left[\frac{\nu}{\theta}+\eta\right] \hat{G}_{s}(z)=z \eta \hat{G}_{c}(z) .
$$

Put in the last expressions $z=1$. Then $G(1)=\hat{G}_{c}(1)+\hat{G}_{s}(1)$ obeys the equation

$$
\frac{\partial}{\partial \theta} G(1)=\left[\frac{\nu}{\theta}+\eta+\frac{1}{\eta}\right] G(1)
$$

whose solution is

$$
G(1)=C^{\prime}(\nu) \theta^{\nu} \exp \int^{\theta}\left(\eta\left(\theta^{\prime}\right)+\eta^{-1}\left(\theta^{\prime}\right)\right) \mathrm{d} \theta^{\prime}
$$

where $C^{\prime}(\nu)$ is a constant.

Using (24), (39), and (40) with $t=1$ one concludes that

$$
\frac{\partial}{\partial \theta} k_{c}=\frac{1 / 2+\rho}{\theta} k_{c}+k_{c} \eta
$$

Solving it and using (42) we finds

$$
k_{c}^{2}=C^{\prime \prime}(\nu) \frac{\theta}{\eta} \exp \int^{\theta}\left(\eta\left(\theta^{\prime}\right)+\eta^{-1}\left(\theta^{\prime}\right)\right) \mathrm{d} \theta^{\prime}
$$

with another constant $C^{\prime \prime}(\nu)$.

Comparison of this expression with (102) proves that

$$
\eta k_{c}^{2}=C(\nu) \theta^{1-\nu} G(1)
$$

To find the constant of proportionality, $C(\nu)$, we compare the both sides of this equation when $\theta \rightarrow 0$.

From Section 4 it follows that for $|\nu|<1 / 2$ and $\theta \rightarrow 0$ the dominant contribution to $G(1)$ is due to $\hat{G}_{c}(1)$ and it is straightforward to check that

$$
C(\nu)=\frac{\cos \pi \nu}{2^{\nu+1} \pi^{3 / 2} \Gamma(1 / 2-\nu)} .
$$

For $|\nu|<1 / 2, C(\nu)>0$ and due to the positivity of $G(1)$ it follows from (105) that $\eta>0$ for all positive $\theta>0$. As the only possible moving singularities of $\eta$ are poles this reasoning proves that $\eta$ is bounded for all $\theta>0$ (the behaviour of $\eta$ at large $\theta$ is fixed by $(173))$. 
The list of solutions of the Painlevé III equation (44) which remain bounded as $\theta \rightarrow \infty$ along the real axis has been presented in [10]. Such solutions form one parameter family parametrized by parameter $\lambda$ which determined the large $\theta$ behaviour of solutions

$$
\eta(\theta) \underset{\theta \rightarrow \infty}{\longrightarrow} 1-\lambda \Gamma(\nu+1 / 2) 2^{-2 \nu} \theta^{-\nu-1 / 2} \mathrm{e}^{-2 \theta} .
$$

Bounded solutions are characterized by the following behaviour at small $\theta$

$$
\eta(\theta) \underset{\theta \rightarrow 0}{\longrightarrow}(2 \theta)^{\sigma} B+(2 \theta) B_{1}+(2 \theta)^{1+2 \sigma} B_{2}+(2 \theta)^{2-\sigma} B_{3}+\mathrm{O}\left(\theta^{2+\sigma}\right)
$$

where $\sigma$ (restricted by inequalities $-1<\operatorname{Re} \sigma<1$ ) is related with $\lambda$ as follows

$$
\sigma=\frac{2}{\pi} \arcsin (\pi \lambda) \text {. }
$$

and coefficients $B_{j}$ are

$$
B_{1}=-\frac{\nu}{(1-\sigma)^{2}}, \quad B_{2}=B^{2} \frac{\nu}{(1+\sigma)^{2}}, \quad B_{3}=\frac{1}{16 B(1-\sigma)^{4}}\left(4 \nu^{2}-(1-\sigma)^{2}\right)
$$

with $B=B(\sigma, \nu)$ being a function of $\sigma$ and $\nu$

$$
B(\sigma, \nu)=2^{-3 \sigma} \frac{\Gamma^{2}((1-\sigma) / 2) \Gamma((1+\sigma) / 2+\nu)}{\Gamma^{2}((1+\sigma) / 2) \Gamma((1-\sigma) / 2+\nu)} .
$$

When $0<\nu<1 / 2$ the solution of the integral equation (1) discussed above corresponds to a particular case of general theory in [10] with $\sigma=1-2 \nu$ (cf. (57), (111), and (73) ). It seems that the case when $-1 / 2<\nu<0$ is missed from the analysis of [10] as $1<\sigma=1-2 \nu<2$. For such choice of $\sigma$ the term $B_{3}$ in (110) is identically zero and the asymptotics (56) represents the two leading terms when $\theta \rightarrow 0$. Though in this case the dominant behaviour at small $\theta$ is linear in $\theta, \eta \rightarrow-\theta /(2 \nu)$, its asymptotics at large $\theta$ is given by the same formula (107) but with value of $\lambda$ obtained by the inversion of (109) (see (73))

$$
\lambda=\frac{\cos (\pi \nu)}{\pi} .
$$

\section{Conclusion}

We demonstrate that special solutions of the integral equation (11) can be obtained from ordinary differential equations (26), (27) or (39), (40) with entering constants calculated using the Painlevé III equation (44) with known asymptotics at small and large arguments. Our finding generalizes the results obtained in [6] for the special case $\nu=0$. An interesting consequence of our investigation is the expression (47) for a solution of the Painlevé III equation with asymptotics given by (56) and (73) as the ratio of two infinite series of spheroidal functions with known coefficients. The positivedefiniteness of the kernel forces this Painlevé III solution to be bounded for all positive arguments thus giving another proof of connection formulae for the Painlevé III equation [10] in a special case. 
Painlevé solution of an integral equation

\section{Acknowledgments}

One of the author (EB) is greatly indebted to A. Its for pointing out the important reference [6].

\section{Appendix A. Series solution of the integral equation}

Let $\mu_{m}$ and $Y_{m}(\gamma)$ be generalized eigenvalues and eigenfunctions of operator (2)

$$
\mu_{m} \int_{0}^{\pi}(\sin \gamma)^{-2 \nu} K(|\cos \beta-\cos \gamma|) Y_{m}(\gamma) \mathrm{d} \gamma=Y_{m}(\beta)
$$

As the symmetrized version of the kernel has the form

$$
\tilde{K}(\beta, \gamma)=(\sin \beta)^{-\nu} K(|\cos \beta-\cos \gamma|)(\sin \gamma)^{-\nu}
$$

eigenfunctions $Y_{m}(\gamma)$ form an orthogonal system of functions

$$
\int_{0}^{\pi}(\sin \gamma)^{-2 \nu} Y_{m}(\gamma) Y_{n}(\gamma) \mathrm{d} \gamma=N_{m} \delta_{m n}
$$

where $N_{m}$ is the normalization constant.

If a function $f(x)$ is expanded into a series of functions $Y_{m}(\gamma)$

$$
f(\cos \gamma)=\sum_{m} a_{m} Y_{m}(\gamma)
$$

then the formal solution of the integral equation (1) is

$$
g(\cos \gamma)=(\sin \gamma)^{-2 \nu-1} \sum_{m=1}^{\infty} \mu_{m} a_{m} Y_{m}(\gamma)
$$

To find explicitly eigenvalues and eigenfunctions of (A.1) it was noted in [1] that the function $K\left(\sqrt{x^{2}+y^{2}}\right)$ plays the role of the Green function of the equation

$$
\left(\frac{\partial^{2}}{\partial x^{2}}+\frac{\partial^{2}}{\partial y^{2}}-\frac{2 \nu}{y} \frac{\partial}{\partial y}-\theta^{2}\right) \Psi(x, y)=0
$$

Therefore the function

$$
\Psi^{(\mathrm{ref})}(x, y)=\int_{-1}^{1} g(t) K\left(\sqrt{(t-x)^{2}+y^{2}}\right) \mathrm{d} t
$$

is a uni-valued solution of (A.6) in all points of the $(x, y)$-plane except the strip $[-1,1]$ which exponentially decays at large distances

$$
\Psi^{(\mathrm{ref})}(r \cos \phi, r \sin \phi) \underset{r \rightarrow \infty}{\longrightarrow} \sqrt{\frac{2}{\theta \pi}} r^{\nu-1 / 2} \mathrm{e}^{-\theta r} \int_{-1}^{1} g(t) \mathrm{e}^{\theta t \cos \phi} \mathrm{d} t
$$


Consider a Dirichlet-type problem of finding the solution of (A.6) in the form

$$
\Psi(x, y)=\Psi^{(\mathrm{inc})}(x, y)+\Psi^{(\mathrm{ref})}(x, y)
$$

where $\Psi^{(\mathrm{inc})}(x, y)$ also obeys (A.6) and the total field $\Psi(x, 0)$ is zero at the interval $[-1,1]$

$$
\Psi(x, 0)=0, \quad-1 \leq x \leq 1 .
$$

It means that unknown function $g(t)$ obeys (1) with $f(x)=-\Psi^{(\text {inc) }}(x, 0)$.

Equation (A.6) permits the separation of variables in the elliptic coordinates

$$
x=\cosh \xi \cos \gamma, \quad y=\sinh \xi \sin \gamma .
$$

It is plain that

$$
\frac{\partial^{2}}{\partial x^{2}}+\frac{\partial^{2}}{\partial y^{2}}=\frac{2}{\cosh 2 \xi-\cos 2 \gamma}\left(\frac{\partial^{2}}{\partial \xi^{2}}+\frac{\partial^{2}}{\partial \gamma^{2}}\right)
$$

and

$$
\frac{\partial}{\partial y}=\frac{2}{\cosh 2 \xi-\cos 2 \gamma}\left(\cosh \xi \sin \gamma \frac{\partial}{\partial \xi}+\sinh \xi \cos \gamma \frac{\partial}{\partial \gamma}\right) .
$$

If $\Psi(x, y)=X(\xi) Y(\gamma)$ then $Y(\gamma)$ and $X(\xi)$ obey

$$
Y_{m}^{\prime \prime}-2 \nu \cot \gamma Y_{m}^{\prime}-\left(\alpha_{m}-\frac{1}{2} \theta^{2} \cos 2 \gamma\right) Y_{m}=0
$$

and

$$
X_{m}^{\prime \prime}-2 \nu \operatorname{coth} \xi X_{m}^{\prime}+\left(\alpha_{m}-\frac{1}{2} \theta^{2} \cosh 2 \xi\right) X_{m}=0 .
$$

The separation constant, $\alpha_{m}$, is chosen to ensure the symmetry properties of solutions

$$
Y_{m}(-\gamma)=Y_{m}(\gamma), \quad Y_{m}(\gamma)=Y_{m}(\gamma+\pi) .
$$

The solution $X_{m}(\xi)$ is fixed by its normalization at infinity

$$
X_{m}(\xi) \underset{\xi \rightarrow \infty}{\longrightarrow} C \mathrm{e}^{\xi(\nu-1 / 2)} \exp \left(-\frac{\theta}{2} \mathrm{e}^{\xi}\right)
$$

where $C$ is a constant.

The above equations are particular cases of spheroidal equation [9]. $Y_{m}(\gamma)$ is even periodic angular solution of (A.14) and $X_{m}(\xi)$ is the radial solution of the modified equation (A.15) decreasing at the infinity. It is known that $Y_{m}$ are orthogonal functions on interval $[0, \pi]$ as in (A.3) and they form a complete set in the space of even functions on this interval.

Therefore, the reflected uni-valued field in the $(x, y)$-plane cut along the interval $[-1,1]$, decaying at infinity (cf. (A.17)), and obeying (A.10) with $f(x)$ as in (A.4) can be represented as a formal series

$$
\Psi^{(\mathrm{ref})}(\xi, \gamma)=\sum_{m} a_{m} \frac{X_{m}(\xi)}{X_{m}(0)} Y_{m}(\gamma)
$$


As for the usual Dirichlet problem the value of $g(t)$ in (A.7) is related with the normal derivative of $\Psi^{(\mathrm{ref})}$ at the strip $[-1,1]$. At small $w$

$$
K_{\nu}(w) \underset{w \rightarrow 0}{\longrightarrow} w^{-\nu} \frac{\Gamma(\nu)}{2^{1-\nu}}+w^{\nu} \frac{\Gamma(-\nu)}{2^{1+\nu}}
$$

As $w=\sqrt{(x-t)^{2}+y^{2}}$ and for $\nu<1 / 2$

$$
\lim _{y \rightarrow 0+} \frac{y^{1-2 \nu}}{\left(x^{2}+y^{2}\right)^{1-\nu}}=\frac{\sqrt{\pi} \Gamma(1 / 2-\nu)}{\Gamma(1-\nu)} \delta(x)
$$

one gets that [1]

$$
g(x)=-\frac{2^{\nu} \theta^{-\nu}}{\sqrt{\pi} \Gamma(1 / 2-\nu)} \lim _{y \rightarrow 0} y^{-2 \nu} \frac{\partial}{\partial y} \Psi^{(\mathrm{ref})}(x, y) .
$$

The behaviour of the decaying solution $X_{m}(\xi)$ at small $\xi$ follows from (A.15)

$$
X_{m}(\xi) \underset{\xi \rightarrow 0}{\longrightarrow} A_{m}+B_{m} \xi^{1+2 \nu} .
$$

For $x$ in the strip $[-1,1]$ and small $y, y=\xi \sin \gamma$. Therefore

$$
g(\cos \gamma)=-\frac{2^{\nu}(2 \nu+1) \theta^{-\nu}}{\sqrt{\pi} \Gamma(1 / 2-\nu)}(\sin \gamma)^{-2 \nu-1} \sum_{m} a_{m} \frac{B_{m}}{A_{m}} Y_{m}(\gamma)
$$

It means that eigenfunctions of operator (A.1) equal $Y_{m}$ and the corresponding eigenvalues are

$$
\mu_{m}=-\frac{2^{\nu} \theta^{-\nu}(2 \nu+1) B_{m}}{\sqrt{\pi} \Gamma(1 / 2-\nu) A_{m}}
$$

where $A_{m}$ and $B_{m}$ are determined from the dominant behaviour at small $\xi$ of the decaying solution of the radial equation $X_{m}(\xi)$ (A.22).

\section{Appendix B. Spheroidal functions}

A convenient method to find periodic functions $Y_{m}(\gamma)$ is to expand them into a series of Gegenbauer's polynomials [9]

$$
Y_{m}(\gamma)=\sum_{n \equiv m \bmod 2} b_{n} C_{n}^{-\nu}(\cos \gamma)
$$

where the summation is performed over all non-negative integers of the same parity as $m$.

Here $C_{n}^{\mu}(x)$ are polynomial solutions of the equation

$$
\left(1-x^{2}\right) y^{\prime \prime}-(2 \mu+1) x y^{\prime}+n(n+2 \mu) y=0
$$

and $C_{n}^{\mu}(\cos \gamma)$ are solutions of

$$
y^{\prime \prime}+2 \mu \cot \gamma y^{\prime}+n(n+2 \mu) y=0 .
$$


We impose the standard normalization [11]

$$
C_{n}^{\mu}(1)=\frac{\Gamma(n+2 \mu)}{n ! \Gamma(2 \mu)}
$$

so the orthogonality relation for Gegenbauer's polynomials is

$$
\int_{-1}^{1}\left(1-x^{2}\right)^{\mu-1 / 2} C_{n}^{\mu}(x) C_{m}^{\mu}(x) \mathrm{d} x=\delta_{n m} \frac{2^{1-2 \mu} \pi \Gamma(n+2 \mu)}{n ![\Gamma(\mu)]^{2}(n+\mu)} .
$$

Notice that [11]

$$
\mu C_{n}^{\mu}(\cos \gamma) \underset{\mu \rightarrow 0}{\longrightarrow} \frac{2}{n} \cos (n \gamma)
$$

so for $\mu \rightarrow 0$ series (B.1) can be transformed to series used for even Mathieu functions. Using the recurrence relation for Gegenbauer polynomials [11]

$$
(n+1) C_{n+1}^{\mu}(x)=2(n+\mu) x C_{n}^{\mu}(x)-(n+2 \mu-1) C_{n-1}^{\mu}(x)
$$

gives

$$
\cos 2 \gamma C_{n}^{-\nu}(\cos \gamma)=A_{n} C_{n}^{-\nu}(\cos \gamma)+B_{n-2} C_{n-2}^{-\nu}(\cos \gamma)+D_{n+2} C_{n+2}^{-\nu}(\cos \gamma)
$$

where

$$
A_{n}=-\frac{\nu(1+\nu)}{(n-\nu)^{2}-1}, B_{n}=\frac{(n-2 \nu+1)(n-2 \nu)}{2(n-\nu+2)(n-\nu+1)}, D_{n}=\frac{(n-1) n}{2(n-\nu-2)(n-\nu-1)} .
$$

Substituting the formal series (B.1) into (A.14) leads to a three-diagonals matrix for the determination of $b_{n}$ with fixed parity

$$
\left[\frac{1}{2} \theta^{2} A_{n}-n(n-2 \nu)\right] b_{n}+\frac{1}{2} \theta^{2} B_{n} b_{n+2}+\frac{1}{2} \theta^{2} D_{n} b_{n-2}=\alpha_{m} b_{n} .
$$

Eigenvalues of the above matrix determine separation constants $\alpha_{m}$.

From (B.5) it follows that the normalization constant for this solution is

$$
N_{m}=\sum_{n \equiv m \bmod 2} b_{n}^{2} \frac{2^{1-2 \mu} \pi \Gamma(n+2 \mu)}{n ![\Gamma(\mu)]^{2}(n+\mu)} .
$$

The function $\mathrm{e}^{\theta x}$ is a solution of (A.6). Therefore it can be expanded in elliptic coordinates A.11) as

$$
\mathrm{e}^{\theta \cosh \xi \cos \gamma}=\sum_{m} Y_{m}(\gamma) \tilde{X}_{m}(\xi)
$$

where $\tilde{X}_{m}(\xi)$ are certain solutions (to be discussed below) of the modified equation (A.15).

Using (A.3) one gets

$$
\tilde{X}_{m}(\xi)=\frac{1}{N_{m}} \int_{0}^{\pi}(\sin \gamma)^{-2 \nu} \mathrm{e}^{\theta \cosh \xi \cos \gamma} Y_{m}(\gamma) \mathrm{d} \gamma
$$


Due to the symmetry properties (A.16)

$$
\tilde{X}_{2 m}(\xi)=\frac{1}{N_{2 m}} \int_{0}^{\pi}(\sin \gamma)^{-2 \nu} \cosh [\theta \cosh \xi \cos \gamma] Y_{2 m}(\gamma) \mathrm{d} \gamma
$$

and

$$
\tilde{X}_{2 m+1}(\xi)=\frac{1}{N_{2 m+1}} \int_{0}^{\pi}(\sin \gamma)^{-2 \nu} \sinh [\theta \cosh \xi \cos \gamma] Y_{2 m+1}(\gamma) \mathrm{d} \gamma
$$

Using Gegenbauer's integral [11]

$$
n ! \int_{0}^{\pi} \mathrm{e}^{\mathrm{i} z \cos \gamma} C_{n}^{\mu}(\cos \gamma)(\sin \gamma)^{2 \mu} \mathrm{d} \gamma=2^{\mu} \sqrt{\pi} \Gamma(\mu+1 / 2) \Gamma(n+2 \mu) \mathrm{i}^{n} z^{-\mu} J_{n+\mu}(z)
$$

one obtains

$$
\tilde{X}_{m}(\xi)=\frac{2^{-\nu} \Gamma(1 / 2-\nu)}{N_{m} \Gamma(-2 \nu)} \sum_{n \equiv m \bmod 2} b_{n} \frac{\Gamma(n-2 \nu)}{n !}(\theta \cosh (\xi))^{\nu} I_{n-\nu}(\theta \cosh (\xi))
$$

where $I_{\mu}(x)$ are modified Bessel functions of the first kind.

These functions increase exponentially at large $\xi$ and the series (B.17) represent a solution of (A.15) of the first kind. As $I_{n-\nu}$ and $(-1)^{n} K_{n-\nu}$ obey the same recurrent relations, the exponentially decaying solutions called solutions of the third kind (denoted in Appendix A by $\left.X_{m}(\xi)\right)$ take the form

$$
X_{m}(\xi)=\frac{2^{-\nu} \Gamma(1 / 2-\nu)}{N_{m} \Gamma(-2 \nu)} \sum_{n \equiv m \bmod 2}(-1)^{n} b_{n} \frac{\Gamma(n-2 \nu)}{n !}(\theta \cosh (\xi))^{\nu} K_{n-\nu}(\theta \cosh (\xi)) .
$$

[1] Belward J A 1969 The solution of an integral equation of the first kind on a finite interval Quart. Appl. Math. 27313

[2] Mkhitaryan S M 1984 A special relationship in spheroidal wave functions and its application to contact problems Prikl. Matem. Machan. 48845

[3] Sommerfeld A 1954 Optics (New York: Academic Press)

[4] Morse P M and Rubenstein P J 1938 The diffraction of waves by ribbons and by slits Phys. Rev. $\mathbf{5 4} 895$

[5] Latta G E 1956 The solution of a class of integral equations J. Rational Mech. Anal. 6821

[6] Myers J M 1965 Wave scattering and the geometry of a strip J. Math. Phys. 61839

[7] Wu T T, McCoy B M, Tracy C A and Barouch E 1976 Spin-spin correlation functions for the two-dimensional Ising model: exact theory in the scaling region Phys. Rev. B 13316

[8] Williams M H 1982 Diffraction by a finite strip Quart. J. Mech. Appl. Math. 35103

[9] Meixner J and Schäfke F W 1954 Mathiusche funktionen und sphäroid functionen (Würzburg: Springer-Verlag)

[10] McCoy B M, Tracy C A and Wu T T 1977 Painlevé functions of the third kind J. Math. Phys. 18 1058

[11] Erdelyi A 1953 Higher transcendental functions v. II ( New York: McGraw-Hill Book Company)

[12] Williams W E 1972 A note on integral equation Glasgow Math. J. 13119

[13] Noble B 1988 Methods based on the Wiener-Hopf thechnique ( New York: Chelsea Publ. Company)

[14] Craster R V, Shanin A V and Doubravsky E M 2003 Embedding formulae in diffraction theory Proc. R. Soc. Lond. A 4592475

[15] Belward J A 1972 Solutions of some Fredholm integral equations using fractional integration, with an application to a forced convection problem J. Appl. Math. Phys. (ZAMP) 23901 